\title{
Evaluation of the effectiveness of hyaluronic acid for the treatment of seborrheic dermatitis of the face
}

\author{
Gagik Hakobyan DMsC*1, PhD', Irina Komissarova ${ }^{2}$, Zoya Evsyukova PhD ${ }^{3}$, Elena Ribakova PhD ${ }^{4}$, Armen Haruthyunyan ${ }^{5}$ \\ ${ }^{1}$ Head of Department of Oral and Maxillofacial Surgery, Yerevan State Medical University after M. Heratsi, Armenia. \\ ${ }^{2}$ Dermatologist, Cosmetologist, Clinica Aesthetic Cosmetology "Limage", Izhevsk, RF. \\ ${ }^{3}$ Dermatologist, cosmetologist, University Clinic of the Russian State Social University, Moscow, RF. \\ ${ }^{4}$ Dermatologist, Cosmetologist, Head of Esthetic Centre Renew, Renew Premium,Moscow, RF. \\ ${ }^{5}$ Plastic Surgon, Astkhik Medical Center, Department of Oral and Maxillofacial Surgery, Yerevan State Medical University after M. Heratsi, Armenia. \\ *Corresponding Author: Gagik Hakobyan, Head of Department of Oral and Maxillofacial Surgery, Yerevan State Medical University after M. \\ Heratsi, 0028 Kievyan str. 10 ap. 65 Yerevan, Armenia.
}

Received date: October 27, 2020; Accepted date: November 27, 2020; Published date: December 07, 2020

Citation: G Hakobyan, I Komissarova, Z Evsyukova, E Ribakova, A Haruthyunyan. (2020) Evaluation of the effectiveness of hyaluronic acid for the treatment of seborrheic dermatitis of the face. Biomedical Research and Clinical Reviews. 1(5); DOI:10.31579/2692-9406/031

Copyright: () 2020 Gagik Hakobyan, This is an open-access article distributed under the terms of the Creative Commons Attribution License, which permits unrestricted use, distribution, and reproduction in any medium, provided the original author and source are credited.

\section{Abstract}

Objectives: The aim of the study was to assess the efficacy of $5 \%$ hyaluronic acid crem for the treatment of seborrheic dermatitis of the face.

Materials and methods: A total of 49 patients (28 men and 21 women, age from 31 to 64 years) with seborrheic dermatitis of the face were selected for the study. A single-site, prospective observational study of $5 \%$ hyaluronic acid cream for the treatment of facial seborrheic dermatitis was conducted from 2016 to 2020.

All patients underwent a thorough clinical examination according to the generally accepted scheme. Treatment planning includes a thorough history and physical examination, preoperative laboratory examination. At each visit, degrees of scale, erythema, and pruritus were evaluated.

To assess the effectiveness of patients were given a questionnaire to fill out the questions. The questionnaire evaluated the $5 \%$ hyaluronic acid crem effectiveness of the treatment.

Outcome was the evolution of the Investigator Global Assessment (IGA) scale, assessing erythema, scale / scaling, seborrhea and pruritus, all measured on a five-point scale, from 0: no signs / symptoms to 4: very severe sign / symptom. Subjects were assessed at baseline after 4 and 8 weeks.

Results: Baseline IGA scores (mean \pm SD) were $9 \pm 3$ (range: 5-13). The use of the EDS significantly reduced the IGA score by $67 \%$ at 4 week and by $83 \%$ at 8 week. The condition improved from baseline in $92.3 \%$ of subjects.

Conclusion: 5\% hyaluronic acid crem has been effective in reducing erythema, scales, seborrhea, and itching. No local side effects were reported.

Keywords: hyaluronic acid; seborrheic dermatitis; erythema; scales

\section{Introduction}

Seborrheic dermatitis of the face is a chronic and recurrent inflammatory skin disease that occurs in areas of the face that are rich in sebaceous gland and is a common dermatological disease worldwide, with a high frequency during puberty and a peak in the third and fourth decades of life [1, 2]. Many factors have been identified as predisposing, and understanding of the causes and pathogenesis has improved due to advances in microbiological and analytical methods [3,4]. The pathogenesis of seborrheic dermatitis is not yet fully understood, but Malassezia yeast, hormones, sebum levels and the immune response are known to play an important role $[5,6]$.

Clinically manifested as erythematous scaly patches or plaques, often associated with itching. Seborrheic dermatitis can affect the head, face, beard, eyebrows, ears or chest. Anywhere we have a proliferation of sebaceous (oil) glands. This inflammatory response additionally disrupts the differentiation of the epidermis and barrier function, and itching and combing can cause further disturbance: as a result, a cycle of immunostimulation, abnormal differentiation of the epidermis and violation of the barrier occur.

The typical symptoms are redness, inflammation, itchiness, flaking skin and thick crusts of buildup. In and in more severe cases sufferers will see yellow scaly pimples. Seborrheic dermatitis can affect the skin of the face, ears, eyebrows, beard, and chest, just like on the scalp.

The goal of treatment should be to provide a safe and effective treatment that improves symptoms, reduces relapses and causes minimal side effects [7]. Because the condition is chronic, treatment should be directed 
at controlling the frequency and severity of flare-ups. Management of seborrheic often requires medical treatment in the form of local antifungal agents, keratolytics, corticosteroids, and calcineurin inhibitors [8].

The treatment facial seborrheic dermatitis often requires drug treatments in the form of topical antifungals, keratolytics, corticosteroids, and calcineurin inhibitors.

Antimicrobial and anti-inflammatory ingredients used, such as zinc, pyroctonolamine, dihydroventantrimide, gum-2 biosaccharide and stearyl glycyrrhetinate, can accelerate the recovery of seborrheic dermatitis and avoid outbreaks $[9,10]$.

Corticosteroids are simple and effective, but they should be used with caution because of their side effects, such as thinning skin and stretch marks, which can occur with prolonged continuous use for weeks or months. Creams or lotions containing the calcineurin inhibitors tacrolimus and pimecrolimus may be effective and cause fewer side effects than corticosteroids, although they are more expensive. Such use is "not for its intended purpose", as they are allowed only for the treatment of atopic dermatitirs. Another reason they are not first-choice drugs is because the seborrheic dermatitis is concerned about a possible cancer link.

Patients often experience anxiety about the cosmetic symptoms of seborrheic dermatitis, so reducing clinical symptoms (erythema, scales, and itching) can help improve any psychological stress caused by the condition. Any concerns regarding the psychological impact of the disease should be addressed using the dermatological quality of life index.

Medical devices include items or substances used to aid in the recognition, prevention, monitoring, treatment, and relief of disease and that achieve this "intended primary effect" by physical, rather than direct pharmacological, immunological, or metabolic means, although such effects may support physical effects.

Hyaluronic acid (HA) plays a multifaceted role in regulating various biological processes and maintaining homeostasis in the body [11].

Hyaluronic acid (HA) is an essential component of connective tissue, skin, vitreous humor, umbilical cord and synovial fluid and plays an important role in maintaining the elasticity and viscosity of the extracellular matrix.

Novel, agents for facial seborrheic dermatitis are a welcome addition to the treatment armamentarium of the dermatologist.
The aim of the study was to assess the efficacy of $5 \%$ hyaluronic acid crem for the treatment of seborrheic dermatitis of the face.

\section{Materials and methods:}

49 patients (28 men and 21 women, age from 31 to 64 years) with seborrheic dermatitis of the face were selected for the study. A single-site, prospective observational study of 5\% hyaluronic acid crem for the treatment of facial seborrheic dermatitis was conducted from 2016 to 2020.

All patients underwent a thorough clinical examination according to the generally accepted scheme. Treatment planning includes a thorough history and physical examination and laboratory examination.

Subjects were instructed to cleanse the affected area with the gentle cleanser and pat dry with a soft towel before applying the provided medication. A thin layer of medication was to be gently massaged in to the affected areas twice daily, in the morning and evening.

The 5\% hyaluronic acid crem was used for four weeks and the purification procedure continued for another four weeks.

The primary outcome was the evolution of the Investigator Global Assessment (IGA) scale, assessing erythema, seborrhea and pruritus, all measured on a five-point scale, from 0: no signs / symptoms to 4: very severe sign / symptom. Subjects were assessed at baseline after 4 and 8 weeks of treatment by an investigator with no knowledge of the type of treatment. Local tolerance was assessed by checking self-reported side effects at each visit.

\section{Results:}

Baseline IGA scores (mean \pm SD) were $9 \pm 3$ (range: $5-13$ ). The use of the $5 \%$ hyaluronic acid significantly reduced the IGA score by $67 \%$ at week 4 and by $83 \%$ at week $8.5 \%$ hyaluronic acid has been effective in reducing erythema, scales, seborrhea, and itching (tabl.1), (figure 1).

\begin{tabular}{|l|l|l|l|}
\hline & Baseline & After 4 weeks & After 8 weeks \\
\hline Scale & 1.9 & 0,8 & 0,6 \\
\hline Erythema & 2.8 & 1.2 & 0.7 \\
\hline Seborrhea & 2.6 & 1.1 & 0.8 \\
\hline Itch & 1.6 & 0.8 & 0.4 \\
\hline
\end{tabular}

Table1: Cinical simthoms patients with seborrheic dermatitis before and after traetment with $5 \%$ hyaluronic acid crem

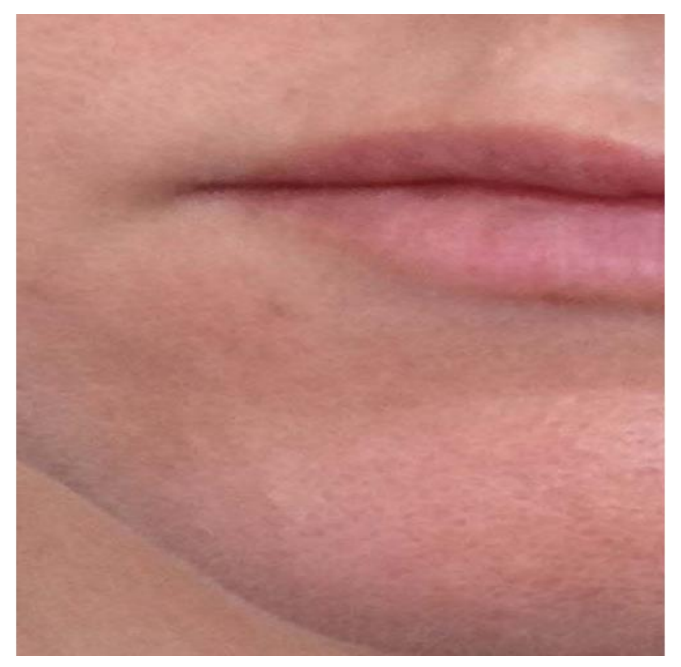




\section{Discusion}

Facial seborrheic dermatitis, characterized by erythema and or flaking/scaling in areas of high sebaceous activity, affects up to five percent of the population.

Despite ongoing study, the cause of the condition is yet unknown, but has been associated with yeast colonization and resultant immune derived inflammation

Facial seborrheic dermatitis is a very common presenting problem in dermatology patients. The papulosquamous disorder is usually found on the scalp, face, or trunk, all of which are sebum-rich areas [13].

The condition is associated with an abnormal immune response caused by a decrease in the titers of antibodies of auxiliary $T$ cells, phytohemagglutinin, stimulation with concanavalin. Multiple factors have been identified as predisposing to facial seborrheic dermatitis, and understanding of the causes and pathogenesis of facial seborrheic dermatitis has improved thanks to advances in microbiological and analytical techniques.

The key factors of proliferation of Malassezia yeast, the state of the epidermis, and high levels of sebum secretion interact, along with other factors, to potentially culminate in facial seborrheic dermatitis.Topical antifungals are another therapeutic mainstay; however, some strains of Malassezia globosa and $M$. restricta, the etiological agents most associated with seborrheic dermatitis, are resistant to azole antifungals, resulting in treatment failure in clinical practice Susceptibility testing of Malassezia species using the urea broth microdilution method [15]. The components break down products that actively stimulate the production of beta-defensin 2 (DEF 32 ) and induce an antibacterial response to allow for healing in the skin's epithelium.

Common therapies often include a topical steroid, which can have untoward effects, such as atrophy, telangiectasias, acne, and perioral dermatitis.

The goal of treatment should be to provide safe and effective treatment that improves symptoms, reduces recurrence, and causes minimal side effects.

There are three main pharmacological treatments for seborrheic dermatitis: keratolytics, anti-inflammatories, and antifungals.

Topical steroids and calcineurin inhibitors reduce inflammation and antifungal agents reduce Malassezia populations. In addition, some agents, such as ketoconazole, have additional anti-inflammatory and antiandrogenic properties. Although these treatments have been demonstrated to be effective, the need for long-term treatment can cause side effects and cosmetic problems that may limit their use. In these cases, the use of topical non-pharmacological therapies may be considered.

Non-pharmacological treatments include cosmetics, cosmeceuticals, and medical devices. The definitions are imprecise, which means that regulations vary from country to country.

Hyaluronic acid (HA), a neutral salt, is a naturally occurring highly conserved polysaccharide found in the skin's tissue.Numerous medical applications, including wound care, have seen improvements as a result of leukocyte, fibroblast, and endothelial cell migration and activation. The efficacy of topical $5 \%$ hyaluronic acid gel on recurrent oral ulcers: comparison between recurrent aphthous ulcers and the oral ulcers of Behçet's disease [15-22].

This study evaluated the efficacy of $5 \%$ hyaluronic acid crem in the treatment of facial seborrheic dermatitis.
Of the parameters, pruritus showed the most pronounced improvement at 8 weeks of treatment. Other parameters also showed improved compared to the baseline, but not as pronounced. A multi-targeted therapy effectivenes whit hyaluronic acidhas anti-inflammatory, sebumcontrolling, and anti-scaling properties is ideal for the management of the signs and symptoms of seborrhea and seborrheic dermatitis could be useful for daily routine use, controlling the symptoms without use of pharmacological therapy

\section{Conclusion:}

In patients diagnosed with facial seborrheic dermatitis use of $5 \%$ hyaluronic acid crem has been effective in reducing erythema, scales, seborrhea, and itching. The condition improved from baseline in $92.3 \%$ of subjects.

\section{Conflict of interest and financial disclosure}

The author declares that he has no conflict of interest and there was no external source of funding for the present study. None of the authors have any relevant financial relationship(s) with a commercial interest.

Consent Statement: Written informed consent was obtained from the patient for publication of this case report and accompanying images.

\section{Funding}

The work was not funded.

\section{Ethical approval}

The study was reviewed and approved by the Ethics Committee of the of the Yerevan State Medical University after M. Heratsi (protocol N16, 5.10.17) and in accordance with those of the World Medical Association and the Helsinki Declaration. Informed consent Patients were informed verbally and in writing about the study and gave written informed consent.

\section{References}

1. Schwartz RA, Janusz CA, Janniger CK. (2006) Seborrheic dermatitis: an overview. Am Fam Physician. 74(1):125-132.

2. Naldi L Seborrhoeic dermatitis.BMJ Clin Evid. 2010;7

3. Karakadze MA, Hirt PA, Wikramanayake TC. (2018) The genetic basis of seborrhoeic dermatitis: a review. J Eur Acad Dermatol Venereol. 32(4):529-536.

4. Bukvić Mokos Z, Kralj M, Basta-Juzbašić A, Lakoš Jukić I. (2012) Seborrheic dermatitis: an update.Acta Dermatovenerol Croat. 20(2):98-104.

5. Dawson TL. (2007) Malassezia globosa and restricta: breakthrough understanding of the etiology and treatment of dandruff and seborrheic dermatitis through whole-genome analysis. J Investig Dermatol Symp Proc. 12(2):15-19.

6. Faergemann J, Borgers M, Degreef HA. (2007) New ketoconazole topical gel formulation in seborrhoeic dermatitis: an updated review of the mechanism.Expert Opin Pharmacother. 8(9):1365-1371.

7. Jaime Piquero-Casals, Doris Hexsel, Juan Francisco MirBonafé, Eduardo Rozas-Muñoz Topical Non-Pharmacological Treatment for Facial Seborrheic Dermatitis.Dermatol Ther (Heidelb). 2019; 9(3): 469-477.

8. Gupta AK, Versteeg SG. (2017) Topical Treatment of Facial Seborrheic Dermatitis: A Systematic Review.Am J Clin Dermatol. 18(2):193-213.

9. Jaime Piquero-Casals,Doris Hexsel, Juan Francisco MirBonafé, Eduardo Rozas-Muñoz. (2019) Topical NonPharmacological Treatment for Facial Seborrheic DermatitisDermatol Ther (Heidelb). 9(3): 469-477. 
10. Li Hui Chen et al. (2018) Hyaluronic acid, an efficient biomacromolecule for treatment of inflammatory skin and joint diseases: A review of recent developments and critical appraisal of preclinical and clinical investigations. International Journal of Biological Macromolecules.116: 572-584.

11. Price RD, Myers S, Leigh IM, Navsaria HA. (2005) The role of hyaluronic acid in wound healing:assessment of clinical evidence. Am J Clin Dermatol. 6 (6):393-402.

12. Sampaio AL, Mameri AC, Vargas TJ, Ramos-e-Silva M, Nunes AP, Carneiro SC. (2011) Seborrheic dermatitis. An Bras Dermatol. 86(6):1061-1071.

13. Borda LJ, Wikramanayake TC. (2015) Seborrheic Dermatitis and Dandruff: A Comprehensive Review.J Clin Investig Dermatol.3 (2).

14. Nakamura $\mathrm{Y}$, Kano R, Murai $\mathrm{T}$, Watanabe S, Hasegawa A. (2000) Antimicrob Agents Chemother. 44(8):2185-2186.

15. Gariboldi $S$ et al. (2008) Low molecular weight hyaluronic acid increases the self-defense of skin epithelium by induction of beta-defensin 2 via TLR2 and TLR4. 181(3):2103-2110.

16. Warshaw E, Wohlhuter RJ, Parneix-Spake A. (2007) Results of a randomized, double-blind, vehicle-controlled efficacy trial of pimecrolimus cream $1 \%$ for the treatment of moderate to severe facial seborrheic dermatitis. J Am Acad Dermatol. 57(2):257264.

17. Lee JH, Jung JY, Bang D. (2008) The efficacy of topical $0.2 \%$ hyaluronic acid gel on recurrent oral ulcers: comparison between recurrent aphthous ulcers and the oral ulcers of Behçet's disease. J Eur Acad Dermatol Venereol. 22 (5):590595.

18. Jiang D, Liang J, Noble PW. (2007) Hyaluronan in tissue and repair. Annu Rev Cell Dev Biol. 23:435-461.

19. Schlesinger TE, Rowland CR. (2012) Efficacy and safety of a low-molecular weight hyaluronic acid topical gel in the treatment of facial seborrheic dermatitis. J Clin Aesthet Dermatol. 5 (10):20-23.

20. Gariboldi S, Palazzo M, Zanobbio L, Selleri S, Sommariva M, Sfondrini L, Cavicchini S, Balsari A, Rumio C. (2008) Low molecular weight hyaluronic acid increases the self-defense of skin epithelium by induction of beta-defensin 2 via TLR2 and TLR4.J Immunol. 181(3):2103-2110.
This work is licensed under Creative Commons Attribution 4.0 License

To Submit Your Article Click Here: Submit Article

DOI: $10.31579 / 2692-9406 / 031$
Ready to submit your research? Choose Auctores and benefit from:

* fast, convenient online submission

* rigorous peer review by experienced research in your field

* rapid publication on acceptance

* authors retain copyrights

* unique DOI for all articles

* immediate, unrestricted online access

At Auctores, research is always in progress.

Learn more www.auctoresonline.org/journals/biomedical-researchand-clinical-reviews- 